\title{
The Effects of DNA Methylation on Medulloblastoma Cell Survivability and Development
}

\author{
Christina Rubino, Anika Chowdhury, Cassandra Greco, Benjamin Honigsfeld, Barbara Pepe, \\ Noelle Cutter*
}

Department of Biological Sciences, Molloy College, Rockville Centre, New York, United States

Email address:

CRubino@lions.molloy.edu (C. Rubino),AChowdhury@lions.molloy.edu (A. Chowdhury), cgreco@lions.molloy.edu (C. Greco), bhonigsfeld@lions.molloy.edu (B. Honigsfeld), BPepe@lions.molloy.edu (B. Pepe), ncutter@molloy.edu (N. Cutter)

${ }^{*}$ Corresponding author

\section{To cite this article:}

Christina Rubino, Anika Chowdhury, Cassandra Greco, Benjamin Honigsfeld, Barbara Pepe, Noelle Cutter. The Effects of DNA Methylation on Medulloblastoma Cell Survivability and Development. International Journal of Biomedical Science and Engineering.

Vol. 8, No. 4, 2020, pp. 51-57. doi: 10.11648/j.ijbse.20200804.12

Received: November 11, 2020; Accepted: December 2, 2020; Published: December 11, 2020

\begin{abstract}
Establish the role epigenetic methylation plays in the segregation of Medulloblastoma subgroups, through pathway analysis in the literature and cancer genome Atlas publicly available data. The goal is to develop an in-vitro assay to study therapeutic responses for subgroups 3 and 4. Functional analysis assays will be used to determine a mechanism for chemoresistance in specific subgroups. In the current study, we assessed Medulloblastoma group 3 (D341) and Group 4 (D283) cell lines using the DNA isolation kit and angiogenesis kits which allows for amplification via PCR and visualize tube formation for tumor nutrients. Twist 1 fended were measured and recorded with the methylation Gold. Group 3 MB cell lines treated with chemotherapy reagents demonstrate altered methylation profiles, angiogenic tube formation increase, a decrease in apoptosis, and underwent phenotypic changes to overcome resistance. Gel electrophoresis results indicated consistent Twist-1 expression in the DAOY+cisplatin cell line in comparison to the control. Cells treated with chemotherapeutic agents evade the mechanisms of apoptosis resulting in angiogenesis. We identified a possible correlation between cisplatin treatment, alteration of methylation patterns, and an increase of Twist-1 gene expression. Twist-1 previously identified to be involved in angiogenesis and cisplatin resistance (Roberts et al. 2016), this supports our hypothesis that alterations in methylation patterns result in an increase in overall chemoresistance.
\end{abstract}

Keywords: Methylation, Angiogenesis, Chemo Resistant, Apoptosis

\section{Introduction}

Medulloblastoma (MB) is the most common malignant brain tumor of childhood, and it accounts for about 20 percent of all childhood brain tumors [23]. Survival rates in children vary depending on the patient's age and how much the tumor spreads. Treatment options for this tumor consist of neurosurgery, craniospinal radiation, and chemotherapy [10]. Unfortunately, many patients become resistant or are intrinsically resistant to treatment [2]. Therefore, a better understanding of the molecular biology of the tumor is essential. There are four subtypes that have been identified in children with medulloblastoma, WNT-activated, SHHactivated, Group 3 (non-WNT / non-SHH), and Group 4 (non-
WNT / non-SHH). The heterogeneous group 3 and group 4 MBs frequently have rare individual genetic alterations, but have been shown to have altered epigenetic regulation, such as distinct methylation profiles. Angiogenesis is feared in cancers such as medulloblastoma. New blood vessels supplying the tumor with blood, oxygen, and nutrients allows the tumor to thrive as well as metastasize. We hypothesize that tumor cells take advantage of alterations in methylation to become resistant to treatment. Our results indicate that Group $3 \mathrm{MB}$ cell lines treated with chemotherapy reagents have altered methylation profiles, avert apoptosis, and undergo phenotypic changes to overcome resistance. Molecularly treatment options are limited, and targeted therapies are still in preclinical development [19]. Therefore, using multiple approaches to attack these aggressive tumors are needed to improve survival 
rates DNA methylation is a biological process in which a methyl group is added to a DNA molecule, changing the way it reacts with other substances, such as transcription factors [16]. DNA methylation also plays a role in gene expression; usually turning genes "off" when the DNA is methylated at those genes [5]. In cancer, such as medulloblastoma, the tumor suppressor genes are most often methylated, or even hypermethylated, which leads to them being turned off. This allows tumors to grow and spread and start forming new blood vessels to supply the tumor with vital nutrients [13].

We focused on the two main subgroups which are group 3 and group 4 [4]. The other two are WNT and SHH, however little is known about the signaling pathways of these two subgroups. WNT is a single transduction signaling pathway that begins with proteins. These proteins pass signals into cells through the surface to the receptors to relay a message. WNT is the least common out of the four subgroups and has the most favorable prognosis [6].

$\mathrm{SHH}$ helps in cerebral development in which it induces proliferation of precursor cells in the developing tissues. $\mathrm{SHH}$ is normally secreted by the purkinje neurons and the tumors are found to arise from the granule neuron precursor cells. Research has shown that there is an overlap in the WNT and SHH signaling pathways making this the second best subgroup for a promising prognosis [24].

Group 3 and Group 4 both have a devastating prognosis and are the most common of the four. Due to the high mortality rates of these subgroups, chemosensitivity is at its highest. Group 3 subgroups are MYC amplified. MYC is a master regulator in the sense that it can transcriptionally activate and repress specific target genes. Group 4 subgroups have a large cell anaplastic histology [24].

\section{Materials and Methods}

\subsection{Cell Lines and DNA Methylation Analysis}

Human medulloblastoma cells were obtained from American Type Culture Collection, ATCC (61381; Manassas, VA, USA) [4, 3]. Cells were grown in the presence of $10 \mathrm{mM}$ glutamine as a supplement in the growth media to render them resistant to chemotherapy. Cells were grown and maintained under humidity at $37^{\circ} \mathrm{C}, 5 \% \mathrm{CO} 2$, and were used for experiments between passages three and six. Confluent cells were harvested using $0.05 \%$ Trypsin-EDTA from Invitrogen (25300-062, Carlsbad, CA). Cells were then collected by centrifugation at $4^{\circ} \mathrm{C}$ for $7 \min (180 \mathrm{X} \mathrm{G} / 1000$ rpm) and were counted using trypan blue dye (Invitrogen) on a hemocytometer. To ensure reproducibility, all experiments were carried out in triplicate.

DNA methylation is a very important functional epigenetic modification of mammalian DNA, which has been implicated in embryonic development, chromosomal stability, transcription, $\mathrm{X}$-chromosome inactivation and complex diseases such as cancers [18]. DNA methylation enables cells to control gene expression in a cell type-specific manner, which further leads to different phenotypes. DNA methylation acts as an epigenetic mechanism used by cells to control gene expression and is essential to understand the roles of DNA methylation in regulating how to develop different cell types [5]. A number of mechanisms exist to control gene expression in eukaryotes, but DNA methylation is a commonly used epigenetic signaling tool that can fix genes in the "off" position or silencing of the genes. The MethylFlash $^{\mathrm{TM}}$ Global DNA Methylation Kit (Zymo Epigentek HTB-185) is a complete set of optimized buffers and reagents to colorimetrically quantify global DNA methylation status by specifically measuring levels of 5methylcytosine $(5-\mathrm{mC})$ in a simplified, "one step" reaction. The kit contains all reagents necessary for the quantification of global DNA methylation. In this assay, DNA is bound to strip-wells that are specifically treated to have a high DNA affinity. This allows global DNA methylation to be directly measured more quickly than other techniques [11].

\subsection{Caspase 3 Assay Kit}

The Caspase 3 assay kit (Thermo-Fisher) provides a simple and convenient means for assaying the activity of caspases that recognize the sequence DEVD. The assay is based on spectrophotometric detection of the chromophore $\mathrm{p}$ nitroaniline ( $\mathrm{p}$-NA) after cleavage from the labeled substrate DEVD-pNA. The p-NA light emission can be quantified using a spectrophotometer or microtiter plate reader at 400 or $405 \mathrm{~nm}$. Comparison of the absorbance of p-NA from an apoptotic sample with an uninduced control allows determination of the fold increase in caspase 3 activity. An increase in absorbance reflects increased caspase 3 activity, indicating more induced apoptosis.

\subsection{MitoRed Assay}

MitoRed (needs catalog \#) is a membrane permeable Rhodamine based dye. This dye localizes in the mitochondria and emits red fluorescence. The interaction of MitoRed with the mitochondria depends on the membrane potential of the mitochondria. The mitochondria can be stained with 20 to 200 $\mathrm{nm}$ of MitoRed. The excitation and emission wavelengths of MitoRed are $560 \mathrm{~nm}$ and $580 \mathrm{~nm}$, respectively. This dye only stains mitochondria in live cells, therefore, increased absorbance equates to increased live cells.

\subsubsection{Trypan Blue Exclusion Assay}

One of the earliest and most common methods for measuring cell viability is the trypan blue staining assay. Trypan blue is an azo dye that is cell membrane impermeable and therefore only enters cells with compromised membranes. Upon entry into the cell, trypan blue binds to intracellular proteins thereby rendering the cells a blue color. The trypan blue staining assay allows for a direct identification and enumeration of live (unstained) and dead (blue) cells in a given population. In this assay, a cell suspension is simply mixed with trypan blue and then visually examined to determine whether cells take up or exclude the dye. In the protocol presented here, a viable cell will have a clear cytoplasm whereas a nonviable cell will have a blue 
cytoplasm.

\subsubsection{DNA Isolation}

DNA was extracted from cellular components according to protocol established in QIAamp DNA mini kit from Qiagen. Concentration verified via the ThermoFisher 2000/2000c nanodrop.

\subsubsection{Amplification Via PCR}

Polymerase Chain Reaction utilized to rapidly duplicate genes via temperature manipulation of hydrogen bonds, RNA primers, and Taq polymerase (New England Biolabs). Samples were prepared in New England Biolabs Master Mix Taq. Primers were optimized for melting temperatures, and PCR was run following the standard conditions: initial denaturation at $95^{\circ} \mathrm{C}$ for thirty seconds, followed by 30 cycles of $95^{\circ} \mathrm{C}$ for thirty seconds, $50^{\circ} \mathrm{C}$ for 30 seconds, and $64^{\circ} \mathrm{C}$ for one minute. Samples were kept at $4^{\circ} \mathrm{C}$ until run on the agarose gel. Primer sequences are as follows: Caspase 3, 5'TGACTGGAAAGCCGAAACTC $3^{\prime}$ and $5^{\prime}$ AGCCTCCACCGGTATCTTCT-3'; Caspase 8, 5'CCGAGCTGGACT TGTGACC $-3^{\prime}$ and 5'CTGCCCAGTTCTTCAGCAAT-3', 5'-GACAAGAATCCGACCAAATGGCAAA-3' and 5'-AAAAGGATCCATGAGAAATCCTTGTGG-3' for BIM, 5'GGCTCAGCTACGCCTTCTC-3' and 5'TCCTTCTCTGGAAACAATGACA-3' for TWIST-1, HPRT, 5' CTTCCTCCTCAGACCGCTTT $-3^{\prime}$ and 5'TTTCCAAATCCTCGGCATAA- $3^{\prime}$ for HPRT, 5'CTCTTCTGCCTGCTGCACTTTG-3' and 5'ATGGGCTACAGGCTTGTCACTC3' for TNFa, 5'-AGA GTC TAT AGG CCC ACC CC-3' and 5'-GCT CGA CGC TAG GAT CTG AC-3'P53, 5'CCAAGGAGATCGACCTGGTCAA3' and 5'GCCGTCAAAACTGTGTGTCCCT-3' for CAV1, and 5'GCTTTGTGTCGCTCTTCCGCAT-3' and 5'TACAGCACGCTGAAGACGCCAA-3' for FZD1. All PCR reactions were performed on CFX-96 Bio-Rad RT System in triplicate and validated by the presence of a single band [21].

\subsubsection{Gel Electrophoresis}

Separation of DNA strands by size via application of an electrical current through an agarose gel. This is utilized to provide a qualitative analysis of gene expression. DNA samples were stained with ethidium bromide and run on a $0.7 \%$ agarose gel at 100 volts for $35-50$ minutes. PCR products were visualized using the UV-light camera and imaged for qualitative analysis.

\subsubsection{Endothelial Tube Forming Assay (in Vitro Angiogenesis)}

The in vitro angiogenesis assay kit tests a cell's ability to form tubes indicating pro-angiogenesis. This assay was used to examine cellular phenotype and formation of capillary precursor structures in our cell lines. Control and treated cells ( 3 x 104) were added onto endothelial cell matrix (ECM) gel obtained from Cell Biolabs (San Diego, CA, USA). The assay was carried out according to the manufacturer's instructions. Cells from each condition were added in triplicate in a 96-well sterile plate and were incubated at $37^{\circ} \mathrm{C}$ for about 18 hours. The growth medium was gently removed, and formed tubular structures were fluorescently labelled with Calcein AM. A Zeiss Axiovert 200 M inverted fluorescent microscope and Axio Vision software 4.6 were used to examine the formed tubes. Obtained images were processed and analyzed using Angiogenesis Analyzer, an ImageJ application. Averaged numbers of branches from at least three images per well from three wells per condition were computed [12].

\section{Results}

Global Increase Methylation status in Medulloblastoma

Methylation has the ability to change the activity of DNA segments without changing the actual sequence. This is accomplished by the methylation of histone bodies, which results in the formation of heterochromatin from euchromatin, inactivating a specific gene or several genes. This is a key factor when it comes to Medulloblastoma because hypermethylation and hypomethylation can provide a significant amount of information regarding prognosis. Given that chemoresistance is a major challenge of all cancers, if we can alter the epigenetics it is our hope to induce a therapy response. As represented in Figure 1, there is a slight increase in the amount of methylation present in DAOY DNA as a result of cisplatin treatment. This suggests that cisplatin treatment results in the alteration of the methylation patterns of the cancer cell DNA. This would allow for the deactivation of certain genes, and the activation of others. Future work will cover results for D348+Cisplatin, D283+Cisplatin, anol+Cisplatin .

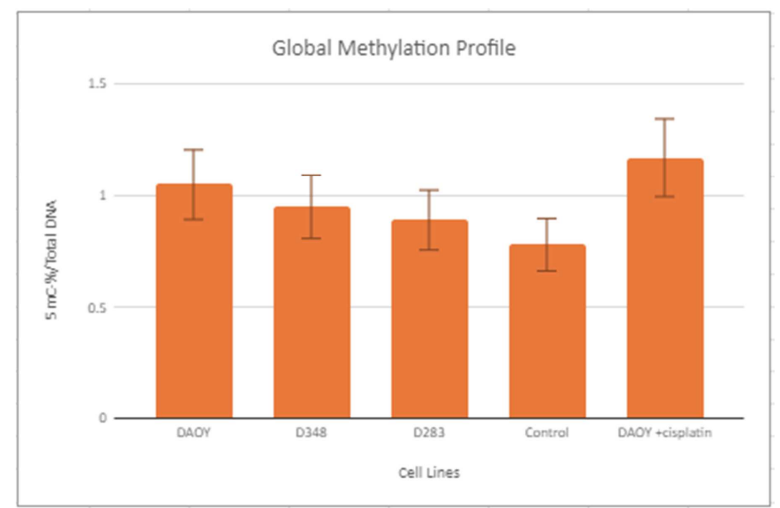

\begin{tabular}{|l|l|}
\hline Cell Line & 5mC-\%/Total DNA \\
\hline DAOY & 1.05 \\
\hline D348 & 0.95 \\
\hline D283 & 0.89 \\
\hline Control & 0.78 \\
\hline DAOY +cisplatin & 1.17 \\
\hline
\end{tabular}

Figure 1. Methylation Pattern Analysis: There is a significant difference between control and DAOY+cisplatin methylation patterns. However, there was no significant difference in global methylation detected among the experimental values. 


\subsection{Apoptosis and Chemo-resistivity}

Apoptosis, otherwise known as programmed cell death, is a growth-limiting regulatory mechanism by which cells can trigger their own death in response to extracellular signals because of irreparable cellular or DNA damage [1]. The process of programmed cell death is especially important in the treatment of cancers, such as Medulloblastoma because chemotherapy is generally the first line of treatment used to induce apoptosis in cancer cells. As shown in Figures 2-5, the MB cell lines are averting the mechanisms of apoptosis despite their treatment with chemotherapeutic compounds in comparison to the control cells. This suggests that the MB cancer cell lines are chemoresistant [22].

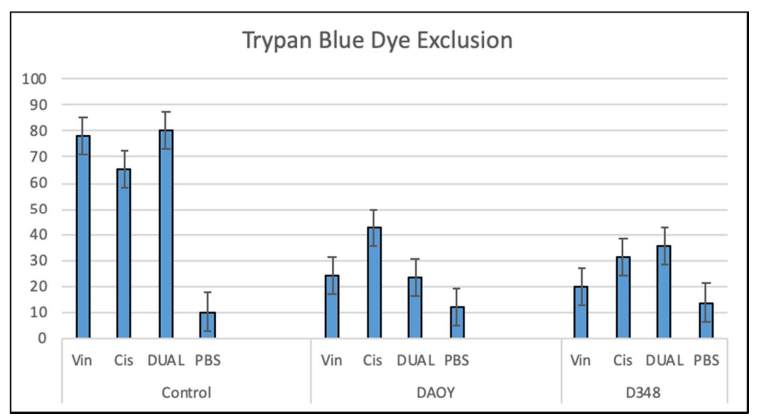

Figure 2. Trypan blue is a dye that only stains cells that are not viable because it cannot cross the plasma membrane in viable cells. The control, $D A O Y$ and D348 cells were all treated with chemotherapeutic agents. After assay, the control cells had a significantly higher amount of cells that were stained blue, this indicates they are undergoing apoptosis while the MB cells lines are evading apoptosis.

Viable cells have cell membranes that are intact. The cell membrane excludes certain types of dyes such as trypan blue from getting into the cell. Cells that do not exclude trypan blue dye, are not viable and therefore, are in an apoptotic state. We treated the control, and cell lines DAOY and D341 with chemotherapeutic compounds. The percentages of the cells that stained blue were much higher for the control group than that of the cell lines. This directly indicates that the control cells are undergoing apoptosis and the cell lines are evading the mechanisms of apoptosis although they were treated with chemotherapeutic drugs.

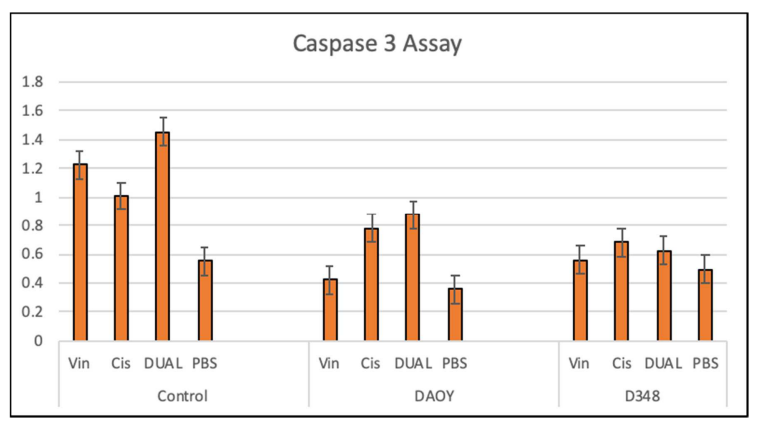

Figure 3. The activity of caspases are detected based upon the spectrophotometric detection of the chromophore p-nitroaniline ( $p$-NA) after cleavage from the labeled substrate DEVD-pNA. After chemotherapeutic based treatment, the average absorbance of $p$-NA light emission was highest for the control. This demonstrates that the $M B$ cell lines are able to avoid the mechanisms of caspases that work to induce apoptosis in cells.

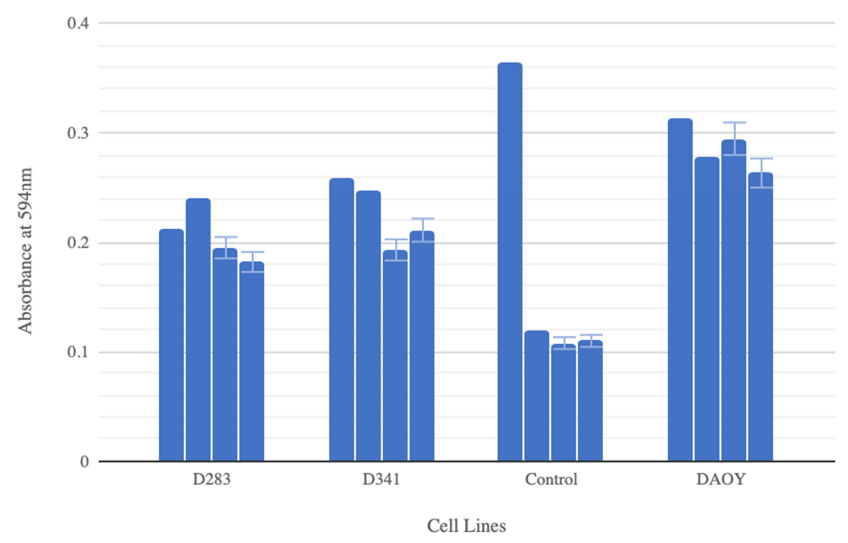

Figure 4. Mito Red is a rhodamine based dye that only stains mitochondria in live cells and emits red fluorescence at an absorbance of $594 \mathrm{~nm}$. After chemotherapeutic treatment the cell lines D283, D341 and DAOY had significantly higher amounts of absorption compared to the control indicating they are averting apoptosis despite chemotherapeutic treatment.

As seen in figure 3 , the activity of caspases that recognize the sequence DEVD was able to be determined using the Caspase 3 assay kit. The activity of caspases are detected based upon the spectrophotometric detection of the chromophore p-nitroaniline ( $\mathrm{p}-\mathrm{NA}$ ) after cleavage from the labeled substrate DEVD-pNA. After chemotherapy based treatment, the average absorbance of p-NA light emission was recorded for the control and the cell lines DAOY and D348. The control cells have the highest amount of absorption, which reflects increased Caspase 3 activity, indicating more induced apoptosis within the cells. Both cell lines treated with chemotherapeutic compounds had a significantly less amount of absorption. This indicated that these cell lines are able to avoid the mechanisms of Caspase 3 that work to induce apoptosis in cells [20].

\begin{tabular}{|l|c|c|c|c|c|c|}
\hline & $\begin{array}{l}\text { \% LIVE (no } \\
\text { treatments) }\end{array}$ & $\begin{array}{l}\text { \% LIVE } \\
\text { Carboplatin }\end{array}$ & $\begin{array}{l}\text { \% LIVE } \\
\text { Cisplatin }\end{array}$ & $\begin{array}{l}\text { \% LIVE } \\
\text { Puro }\end{array}$ & $\begin{array}{l}\text { \% LIVE } \\
\text { DOXO }\end{array}$ & $\begin{array}{l}\text { \% LIVE } \\
\text { Stauro }\end{array}$ \\
\hline Control Average Trials: & 90.3 & 56.8 & 45.8 & 75.6 & 40.2 & 56.8 \\
\hline DAOY & 89.9 & 82.5 & 77.6 & 84.9 & 73.5 & 79.1 \\
\hline D241 & 85.7 & 78.9 & 85.1 & - & - & - \\
\hline D348 & 81.4 & 84.3 & 80.7 & - & - & - \\
\hline
\end{tabular}

Figure 5. Quick and easy two-color assay to determine viability of cells in a population based on plasma membrane integrity and esterase activity. Live cells are distinguished by the presence of ubiquitous intracellular esterase activity, determined by the enzymatic conversion of the virtually nonfluorescent cell-permeant calcein AM to the intensely fluorescent calcein. The polyanionic dye calcein is well retained within live cells, producing an intense uniform green fluorescence in live cells.

Figure 4 Mito Red is a cell membrane permeable Rhodamine-based dye. This dye localizes within mitochondria and emits red fluorescence. The interaction of Mito Red within the mitochondria of the cell is dependent upon the membrane potential of the mitochondria. This dye only stains mitochondria in live cells, therefore an increased absorbance equates to an increased amount of live cells. At an absorbance of $594 \mathrm{~nm}$, the control cells that were treated with chemotherapy drugs had a decreased level of absorption. However, when the cell lines D283, D341 and DAOY were 
treated, the absorbance was much higher than those of the treated control cells. Each of the results on treatments of the cell lines affirmed that cancer cells are evading apoptosis, even when treated with chemotherapeutic compounds.

\subsection{Angiogenesis with the Use of Cisplatin}

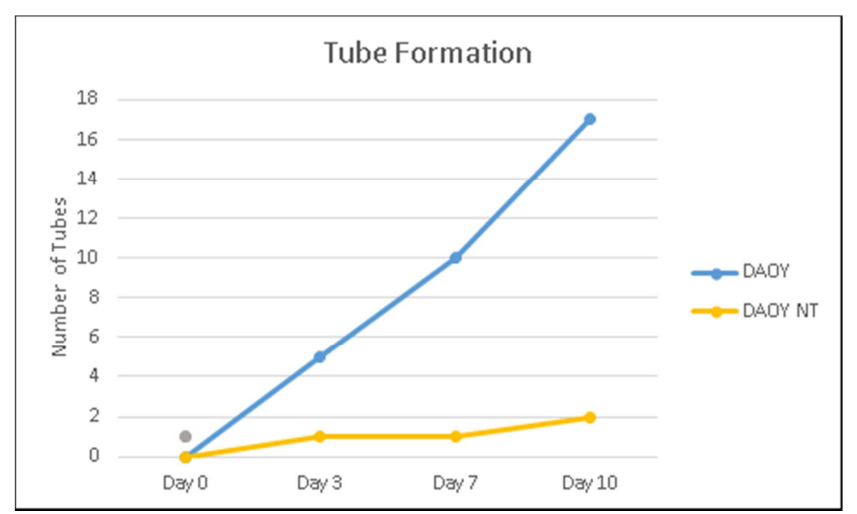

Figure 6. This chart measures tube formation in comparison to the number of days with the use of cisplatin. DAOY NT were used as a control which showed no significant change at Day 10 in tube formation. DAOY cells were treated with cisplatin and presented with a large increase in tube formation.

The formation of new blood cells, angiogenesis, is a way that the body supplies the tumor with an adequate amount of nutrients to allow the cell to survive [7]. We used DAOY cells that were treated with cisplatin as well as our control, DAOY NT (no treatment). As seen in figure $3 \mathrm{a}$ and $3 \mathrm{~b}$, angiogenesis increases with the use of cisplatin. As seen over a 10 day period, angiogenic responses increase in the DAOY cell treated with cisplatin, as days continue. This is most likely a result of the tumor trying to survive, by increasing the amount of nutrients to the tumor [17].

Tube formation is a response to the cisplatin. As seen in figure 6 , tube formation increases with the use of cisplatin. This auto response is for tumor growth and survival DAOY + cis DAOY NT.
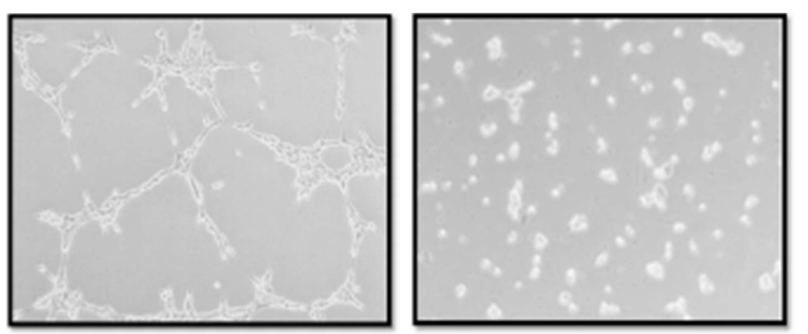

Figure 7. Is showing DAOY with the addition of cisplatin to the left and to the right are the DAOY cells without treatment. Tube formation is presented in the DAOY+Cis in response to the chemotherapy.

\section{Discussion}

There was no significant correlation between the experimental methylation values as we had hypothesized. However, this does not necessarily indicate constant methylation patterns. It is entirely possible that the overall net amount of heterochromatin remained the same, but equal amounts of methylation and acetylation occurred in different locations. Thus, it is still possible that alterations in methylation patterns have occurred. In order to verify this, future research should include additional methylation testing for reliability.

Apoptosis, or programmed cell death, is a growthlimiting regulatory mechanism by which cells can trigger their own death in response to extracellular signals because of irreparable cellular or DNA damage [1]. The ability of tumor cells to elude apoptosis is a hallmark of most types of cancer.

Utilizing the amplified DNA segments generated via PCR, our gel electrophoresis results clearly indicate a difference in the gene expression between our control and DAOY cell lines. In figure 3, p53 and BIM are clearly expressed in our untreated control cell line, as expected in a healthy cell. Both genes are vital in certain apoptotic pathways ensuring the death of a cell too damaged to safely reproduce (NIH, 2019)(Gogda et al. 2013). However, both experimental cell lines DAOY and DAOY+Cisplatin, lack p53 and BIM. Instead, they both express CAV1. This gene is responsible for the production of an integral protein and is commonly found to be overexpressed in metastatic drug-resistant tumors and cancer cell lines (Shatz and Liscovitch, 2008). These findings support the claim that cancer cells and somatic cells display different epigenetic properties.

Furthermore, cisplatin treatment resulted in clear differences in the gene expression within the same cell lines. With respect to our control, there is a loss of BIM expression, in conjunction with increased expression of TNFAI, CASP3, and HPRT. The loss of BIM upon treatment of the control cells was unexpected, as BIM activity has been correlated with neuron apoptosis (BCL2L11 Gene 2020). However, further evidence also suggests that BIM has anti-apoptotic capabilities (Gogada et al. 2013). Therefore, it is possible that BIM was repressed as an anti-apoptotic response, but this requires further investigation. TNFA1 manages a significant amount of processes, including cell proliferation, differentiation, apoptosis through cytokine signalling, thus making it an excellent candidate for observation. CASP3 is responsible for the production of the "executioner" caspase 3 protein, which plays a key role in apoptosis through protein catabolism (Walsh et al. 2008). Finally, HPRT was designated as our negative control, as it's protein product is involved in purine formation, not cell survival or proliferation (HPRT-1 Gene 2020). However, it is possible that there is some novel interaction with cisplatin that we were not anticipating.

When the DAOY cell line was treated with cisplatin, CAV1 remained expressed, while Twist-1 also became expressed. Given the role of Twist-1 in cisplatin resistance and angiogenesis (Roberts et al. 2016), the increased expression of this gene following cisplatin treatment is noteworthy. Our hypothesis is partially confirmed in that our data shows tumor cells are experiencing alterations in gene expression in response to cisplatin to improve chemoresistance through increased cell survivability and 
angiogenesis. A slight change in methylation patterns are demonstrated between DAOY and DAOY+Cpt, but it is not significant. However, it is entirely possible that certain genes were methylated and others were acetylated, resulting in no significant net change in methylation pattern, but still allowing for new genes to be expressed. As stated earlier, additional tests are needed to determine if methylation is responsible for the change in gene expression. Analysis of experimental cell lines D283, D341 (subgroups 3 and 4) are currently pending at this time. In the future, we seek to verify the observed differences in gene expression through repetition of the DNA isolation, PCR and gel electrophoresis procedures.

As part of a survival mechanism, it is demonstrated that DAOY cells treated with cisplatin result in altered hypervascularization phenotype, consistent with angiogenesis, compared to their chemo sensitive counterparts. These results suggest that angiogenesis is a possible mechanism of chemoresistance in MB through the development of a capillary support network which is vital for cancer cells because these blood vessels supply tumor cells with the nutrients for growth. The endothelial tube formation allows a network or capillaries to form within and surrounding the tumor for an adequate supply of blood to the tumor [8].

\section{Conclusion}

Group 3 MB cell lines treated with chemotherapy reagents have altered methylation profiles, avert apoptosis, and undergo phenotypic changes to overcome resistance. It is seen from our results that

Trypan Blue, Caspase 3, MitoRed, Live/Dead, are all assays that are used to determine cell survival and proliferation after treatment with chemotherapeutic compounds. The results from all of these assays imply that the cell lines studied in each of the assays performed, are able to evade the mechanisms of apoptosis, while the control cells were undergoing apoptosis. These cell lines have the ability to thrive even though they were treated with chemotherapeutic drugs. This implicates that these cells have developed chemoresistance [9]. We suggest that chemoresistance displayed by these cell lines is due to the altered methylation profiles, and changes in gene expression. This suggests that cisplatin treatment results in the alteration of the methylation patterns of the cancer cell DNA. This would allow for the deactivation of certain genes, and the activation of others.

Tube formation of capillary networks supply the tumor with oxygen and nutrients to survive harsh chemotherapy treatments. We can suggest that angiogenesis is a mechanism of chemoresistance because of this blood supply. As we treat the cells with Cisplatin, the body in a form of defense to the chemo will supply the tumor with more blood, making medulloblastoma tumors chemo-resistant. These results suggest that angiogenesis is a possible mechanism of chemoresistance in $\mathrm{MB}$.

\section{Declaration of Conflicting Interest}

The authors declare no conflict of interest.

\section{Funding}

This research was supported by the Biology, Chemistry, and Environmental Studies department and through the National Science Foundation grant ID \# 1626093.

\section{References}

[1] Ana S. Guerreiro, Sarah Fattet, Barbara Fischer, Tarek Shalaby, Shaun P. Jackson, Simone M. Schoenwaelder, Michael A. Grotzer, Olivier Delattre and Alexandre Arcaro. (2008) "Targeting the PI3K p110 $\alpha$ Isoform Inhibits Medulloblastoma Proliferation, Chemoresistance, and Migration.” DOI: 10.1158/1078-0432.CCR-08-0385.

[2] Clinical Cancer Research, American Association for Cancer Research (2008). Volume 14, Issue 21, pages 6761- 6769.

[3] "Daoy (ATCC® HTB-186 ${ }^{\mathrm{TM}}$ )." ATCC: The Global Bioresource Center, ATCC, www.atcc.org/products/all/HTB186.aspx\#generalinformation.

[4] "D341 Med (ATCC $®$ HTB-187TM)." ATCC: The Global Bioresource Center, ATCC, www.atcc.org/products/all/HTB187.aspx\#generalinformation.

[5] Edward C. Schwalbe, Daniel Williamson, Janet C. Lindsey, Dolores Hamilton, Sarra L. Ryan, Hisham Megahed, Miklos Garami, Peter Hauser, Bozena Dembowska-Baginska, Danuta Perek, Paul A. Northcott, Michael D. Taylor, Roger E. Taylor, David W. Ellison, Simon Bailey, and Steven C. Clifford (2013). "DNA methylation profiling of medulloblastoma allows robust sub-classification and improved outcome prediction using formalin-fixed biopsies." Acta neuropathologica; 125 (3): 359-371. doi: 10.1007/s00401-0121077-2.

[6] Frič, R., Due-Tønnessen, B. J., Lundar, T. et al. Long-term outcome of posterior fossa medulloblastoma in patients surviving more than 20 years following primary treatment in

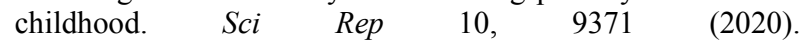
https://doi.org/10.1038/s41598-020-66328-8.

[7] “HCN-2 (ATCC® CRL-10742 $\left.{ }^{\mathrm{TM}}\right) . "$ ATCC: The Global Bioresource Center, ATCC, www.atcc.org/Products/All/CRL10742.aspx\#generalinformation.

[8] Hillen, F.; Griffioen, A. W. (2007). Tumour vascularization: Sprouting angiogenesis and beyond. Cancer Metastasis Rev., 26, 489-502.

[9] Housman, G.; Byler, S.; Heerboth, S.; Lapinska, K.; Longacre, M.; Snyder, N.; Sarkar, S. (2014) Drug resistance in cancer: An overview of Cancers, 1769-1792.

[10] Huang, M. A., MD, Barbour, M., MD, Sakamoto, K. M., MD, PHD, MacDonald, T. J., MD, \& Packer, R. J., MD. (2018, April 23). Pediatric Medulloblastoma Treatment \& Management. https://reference.medscape.com/article/987886treatment\#d5Infinium MethylationEPIC Kit. (2018).

[11] https://www.illumina.com/products/by-type/microarraykits/infinium-methylation-epic.html. 
[12] Protein Biology Resource Library. Overview of Western Blotting, 2018. https://www.thermofisher.com/us/en/home/lifescience/protein-biology/protein-biology-learningcenter/protein-biology-resource-library/pierce-proteinmethods/overview-westernblotting.html.

[13] Jin, Z., \& Liu, Y. DNA methylation in human diseases. Genes \& Diseases, 2018; 5 (1), 1-8. doi: https://doi.org/10.1016/j.gendis.2018.01.002.

[14] Laederich, Melanie. "The Top 10 Western Blotting Mistakes (and Solutions!).” Bitesize Bio, Science Squared, 2017.

[15] bitesizebio.com/19799/the-top-10-western-blotting-mistakesand-solutions/.

[16] National Cancer Institute Center for Cancer Research. Medulloblastoma. (2018).

[17] Polkinghorn WR, Tarbell NJ. "Medulloblastoma: tumorigenesis, current clinical paradigm, and efforts to improve risk stratification." Nat Clin Pract Oncol 2007; 4: 295-304.
[18] Razin, A., \& Cedar, H. (1991). DNA methylation and gene expression. Microbiological Reviews, 55 (3), 451-458.

[19] Sengupta, S., Pomeranz Krummel, D., \& Pomeroy, S. (2017). The evolution of medulloblastoma therapy to personalized medicine. F1000Research, 6, $490 . \quad$ doi: 10.12688/f1000research.10859.1.

[20] Tamayo-Orrego, L.; Chia-Lun, W.; Bouchard, N.; Khedher, A.; Swikert, S.; Remke, M.; Skowron, P.; Taylor, M. (2016) Charron. F.; Evasion of cell senescence leads to medulloblastoma progression. Cell Reports, 14, 2925-2937.

[21] Vibhakar, R., Foltz, G., Yoon, J. G., Field, L., Lee, H., Ryu, G. Y., Pierson, J., Davidson, B., ... Madan, A. (2007). Dickkopf1 is an epigenetically silenced candidate tumor suppressor gene in medulloblastoma. Neuro-oncology, 9 (2), 135-44.

[22] Zheng, H. The molecular mechanisms of chemoresistance in cancers. Oncotarget (2017), 8, 59950-59964.

[23] http://cancerres.aacrjournals.org/content/58/5/896.full-text.pdf.

[24] https://www.ncbi.nlm.nih.gov/pubmed/10564585. 